\title{
Designing large-scale antenna array using sub-array
}

\author{
Naimul Mukit, Md. Rafiqul Islam, Mohamed Hadi Habaebi, A. H. M. Zahirul Alam, Khaizuran \\ Abdullah, Norun Farihah Abdul Malek, Rauful Nibir, Noor Hidayah M. Adnan, Eid Osman \\ Department of Electrical and Computer Engineering, Faculty of Engineering, \\ International Islamic University Malaysia, Malaysia
}

\begin{tabular}{l} 
Article Info \\
\hline Article history: \\
Received Jan 11, 2019 \\
Revised May 13, 2019 \\
Accepted May 28, 2019 \\
\hline
\end{tabular}

Keywords:

Large antenna array

Scalability

Sub-array

\begin{abstract}
Antenna array of large scale have been examined for different applications including 5G technology. To get better data rate or a reliable link substantial number of antenna arrays have been utilized to provide high multiplexing gains as well as array gains with high directivity. In this paper a simple but efficient implementation technique of using sub-arrays for the improvement of large-sized uniform arrays. By repeating a small sub-array multiple times large arrays can be designed. This implication of utilizing small array simplifies the design of a larger array which allows the designer to concentrate on the smaller sub-array before assembling larger arrays. So, by investigating the sub arrays the performance and radiation characteristics of large arrays can be anticipated. The array-factor for a planar sub-array of 2x2 (4 elements) is analyzed using Mat-lab software and then a large array is formed by placing the $2 \times 2$ sub-array in different configurations in a rectangular arrangements up to $8 \times 8$ planar array. And then the results are validated with CST (Computer simulation technology) simulation results. In this way the array-factors, directivities, HPBWs, and side lobes of the constructed large arrays are analyzed and associated with the small sub-array.
\end{abstract}

Copyright $@ 2019$ Institute of Advanced Engineering and Science. All rights reserved.

\section{Corresponding Author:}

Md. Rafiqul Islam,

Department of Electrical and Computer Engineering,

Faculty of Engineering, International Islamic University Malaysia,

53100 Kuala Lumpur, Malaysia.

Email: rafiq@iium.edu.my

\section{INTRODUCTION}

Large-Scale Antenna Array (also known as Massive MIMO or Hyper MIMO) system is a vital notion in wireless communications research areas, which highlights the importance of advanced 5G technological network architectures. At the base stations a huge number of array antennas operate consistently in order to direct the signals into smaller regions of space. Additionally, two or more antennas is outfitted with the consumer gadgets to facilitate the reception of signals, consequently there is a considerable enhancement in electricity effectivity as well as the throughput [1-4]. As large antenna arrays generally resonate in millimeter wave frequency ranges, their position can be confined in a very small area with a variety of array configurations i.e. planar, circular, spherical or cylindrical [5-6].

The configuration of the array placement, the range of elements, the characteristics of the radiation pattern, the inter component spacing and mutual coupling amongst the array factors each make contributions in the ultimate performance of any massive MIMO system. The consistent frequencies that are used for Massive MIMO structures are 3-6GHz, 27-28GHz and $60-70 \mathrm{GHz}$, whilst the kind of antennas investigated can be shaped as patch antennas, horn antennas and also dipoles in some cases. In Figure 1, a $2 \times 2$ rectangular antenna array configuration is used in cell phones as shown. Large planar arrays such as $4 \times 4,8 \times 8,16 \times 16$, and $32 \times 32$ can additionally be geared upinto small areas of less than one square centimeter at the base stations. Planar arrays are characterized with high gains and very directive beams in the preferred directions whilst 
displaying low side lobes in undesired directions. Moreover, as the gain and directivity of an antenna are related to each-other, the array factor can be effortlessly calculated from that relation [7-9].

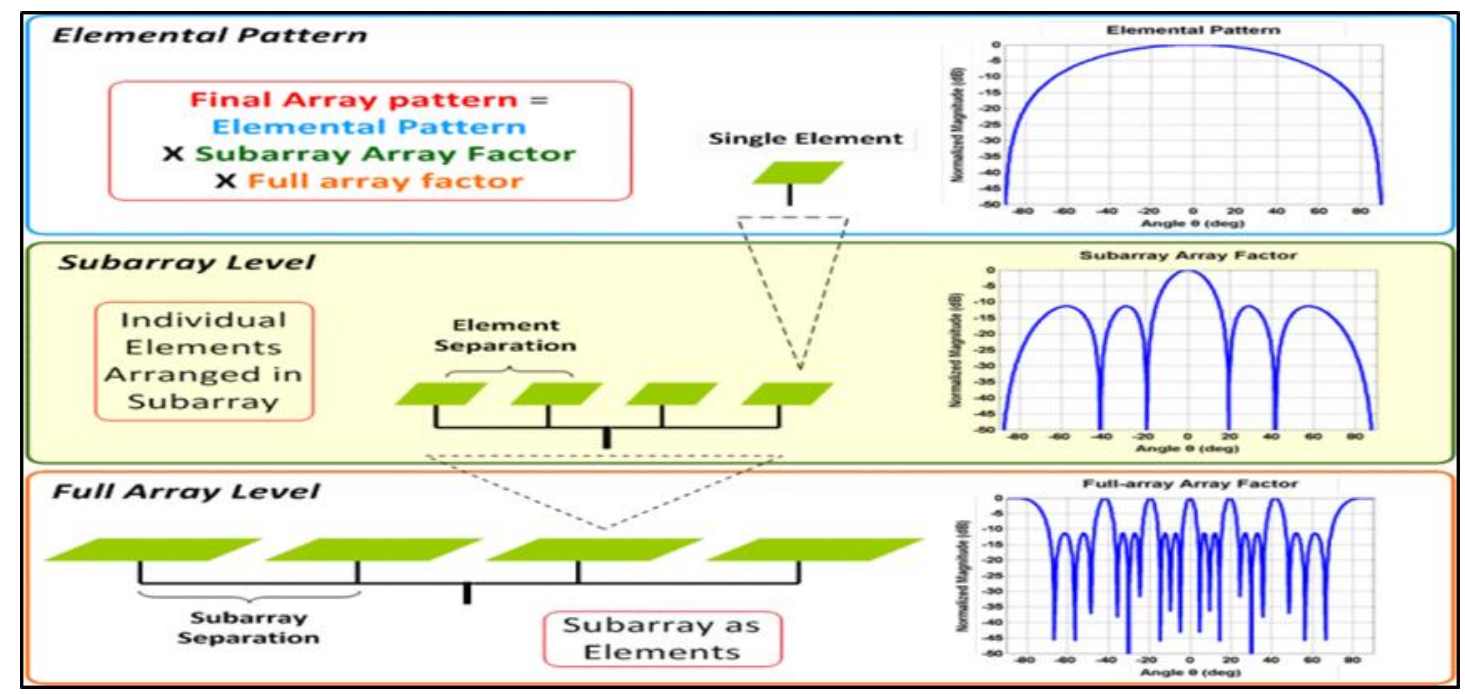

Figure 1. Array synthesis when using sub-array concept [9]

There are quite a few techniques amongst the researchers have been improvised in order to study the characteristics and behavior of massive antenna arrays radiation patterns. One of the most famous strategies of them is used in this venture is the notion of sub-arrays. Sub-arrays are regarded as factors in a large array. This provides an easier analysis as the designer will focus on the small sub-array design and construct large arrays by repeating those sub-arrays throughout the entire large array. According to [10-12], the overall pattern of an array arises from the multiplication of individual element pattern and the array factor. When sub-arrays are involved, the overall pattern can be achieved from the product of element pattern, the subarray factor, and the full array factor determined by the amplitude of excitation, spacing between the elements as well as phasing between sub-arrays.

Sub-array implementation is a very beneficial technique for designing massive array antennas. It simplifies the design by allowing the large array designer to concentrate on the smaller subarray design first and finally use the sub-array element as a part of any other larger array. Without indulging into full array study, this new approach implements the sub-array to construct a larger array. This process reduces large array problems into small array solution which has the potential to save a lot of CPU time and requirement of heavy memory as well. Generally, radiation characteristics of any large array can be easily predicted if the mutual coupling is ignored between the elements of array antenna. By implementing pattern multiplication approach of the array factor as well as the radiation pattern of single element would be adequate to forecast the array radiation performance and characteristics [13-15].

There are many other widely used numerical approaches available for large array analysis. Some of the analysis are based on infinite-array approach as well as the periodicity of the structure while others depend on the actual finite size array. Infinite array method ignores the edge effects while the simulation of the second approach is highly computational, complex and expensive when it comes to large array size. Research mentioned in [9] validates that subarrays can be used as a building block to facilitate the design of large antenna arrays. By controlling the subarray, the overall radiation pattern can be easily predicted. This can be achieved by multiplying the element pattern, sub-array factor and the overall array factor. Figure 1 represents the levels of array factor synthesis.

Any changes that may take place regarding the pattern of the elements of the array factor, due to that change subarray or full array factor will also be hugely affected in the final outcome of the array pattern. Changes can appear in the form of maximum (grating) lobes, variation of side-lobe levels or any other change into symmetrical dysfunction of the array pattern. Grating lobes appear due to distortions in the subarray which results from feeding errors or mutual coupling between feedline and elements. The concept proposed in this paper was the design of $1 \times 16$ array from four $1 \times 4$ sub-arrays. The sub-arrays are manipulated in order to suppress the grating lobes by using parallel and series excitations. 


\section{PROPOSED SUB-ARRAYED PLANAR ARRAYS}

The array factor analysis of $\mathrm{M} \times \mathrm{N}(\mathrm{M}=2,4,8,16,32)$ and $(\mathrm{N}=2,4,8,16,32)$ uniform broadside planar arrays which consists of an isotropic radiators are proposed in order to investigate the radiation characteristics of large-scale arrays. Figure 2 shows the conceptual diagram of describing this arrangement. Starting with $2 \times 2$ (4 element) planar array as a sub-array, a large planar array is formed up to $16 \times 16$ (256 elements). For a planar uniform array of $\mathrm{M} \times \mathrm{N}$ elements in which all elements have same amplitude excitations, the normalized array factor is found as [3, 6-7]:

$$
(\theta, \emptyset)=\left\{\frac{1}{M} \frac{\sin \left(M \frac{\psi_{x}}{2}\right)}{\sin \left(\frac{\psi_{x}}{2}\right)}\right\}\left\{\frac{1}{N} \frac{\sin \left(N \frac{\psi_{y}}{2}\right)}{\sin \left(\frac{\psi_{y}}{2}\right)}\right\}
$$

where, $\psi_{x}=k d_{x} \sin \theta \cos \phi+\beta_{x}$

$$
\psi_{y}=k d_{y} \sin \theta \sin \phi+\beta_{y}
$$

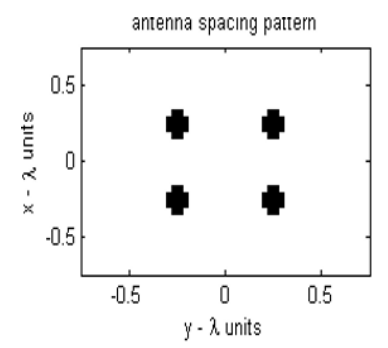

(a)

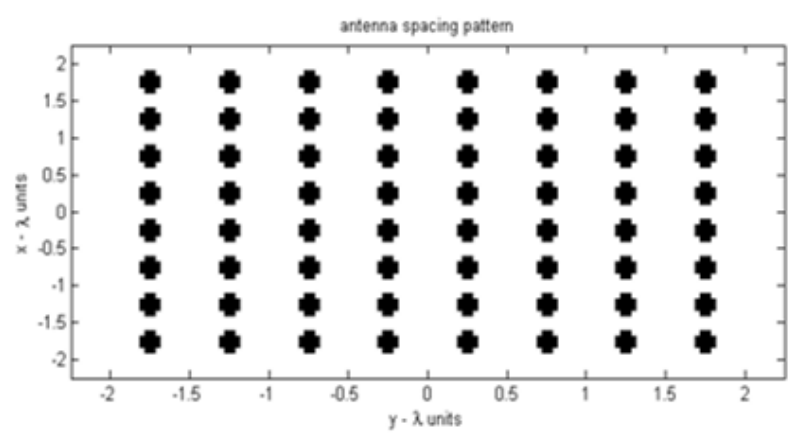

(c)

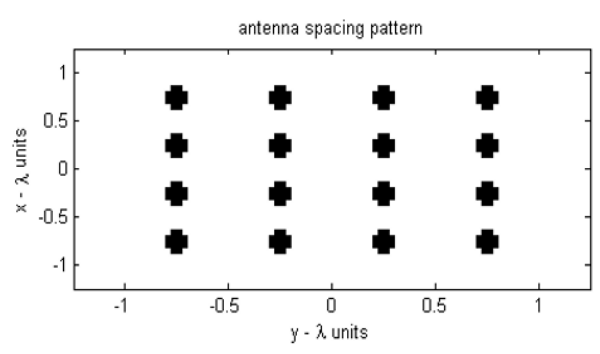

(b)

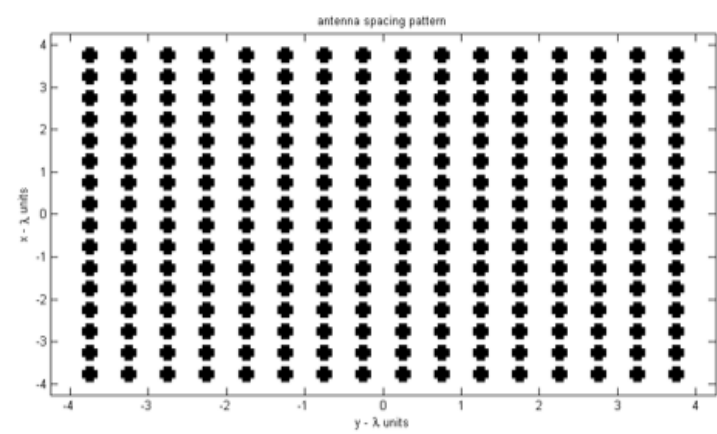

(d)

Figure 2. (a) 2x2, (b) 4x4, (c) 8x8, (d) 16x16 planar array arrangement

The beam solid angle of planar array can be obtained as:

$$
\Omega_{A}=\theta_{h} \phi_{h}
$$

or

$$
\Omega_{A}=\frac{\Delta \theta_{x} \Delta \theta_{y}}{\cos ^{2} \theta_{0} \sqrt{\left[\sin ^{2} \phi_{0}+\frac{\Delta \theta^{2} y}{\Delta \theta^{2} x} \cos ^{2} \phi_{0}\right]\left[\sin ^{2} \phi_{0}+\frac{\Delta \theta^{2} x}{\Delta \theta^{2} y} \cos ^{2} \phi_{0}\right]}}
$$

where $\theta_{0}$ and $\phi_{0}$ specify the direction of the main beam, $\Delta \theta_{x}$ is the half-power beam width (HPBW) of a broadside linear array where $\mathrm{M}$ number of elements and excitation of amplitude is same as the $\mathrm{x}$-axis linear arrays forming the rectangular planar array while $\Delta \theta_{y}$ is the half-power beam width (HPBW) of a broadside linear array where $\mathrm{N}$ number of elements and excitation of amplitude is same as the $\mathrm{y}$-axis linear arrays forming the planar array. 
Directivity is defined as the ratio of maximum power density to its average value over a sphere. This shows how much the antenna is directive compared to isotropic antenna which has a directivity of equal to 1 . To calculate the directivity of an array, the general expression shown in (4) is used [7].

$$
D_{0}=4 \pi \frac{\left|A F\left(\theta_{0}, \phi_{0}\right)\right|^{2}}{\int_{0}^{2 \pi} \int_{0}^{\pi}\left|A F\left(\theta_{0}, \phi_{0}\right)\right|^{2} \sin \theta d \theta d \phi}
$$

For large broadside planar arrays, (4) reduces to (5)

$$
D_{0}=\pi D_{x} D_{y} \cos \theta_{0}
$$

where, $D_{x}$ is the directivity of linear array placed in x-axis whereas $D_{y}$ is the directivity of the second linear array in $y$-axis. Moreover, the array solid beam angle $\Omega_{A}$ in (3) can also be used to find the approximate directivity of large broadside planar arrays as seen in (6).

$$
D_{0}=\frac{\pi^{2}}{\Omega_{A[S r]}} \simeq \frac{32400}{\Omega_{A\left[\operatorname{deg}^{2}\right]}}
$$

where $\Omega_{A}$ is mostly expressed in square degrees or square radians. The gain is calculated as:

$$
G=e r D
$$

So the gain is also defined as the ratio of intensity in a particular direction to the power gain of the reference antenna in its referenced direction.

\section{RADIATION PATTERN ANALYSIS OF SUB-ARRAYED PLANAR ARRAYS}

Based on the Array factor formula given in (1-7), a mat-lab code is created to plot the $\mathrm{NXN}(\mathrm{N}=2,4$, $8,16,32) 2 \mathrm{D}$ array factor plots starting with $2 \times 2$ (4 element) planar array as a sub-array and then a large planar array is formed up to 16x 16 (256 elements). The plots are as shown in Figure 3.
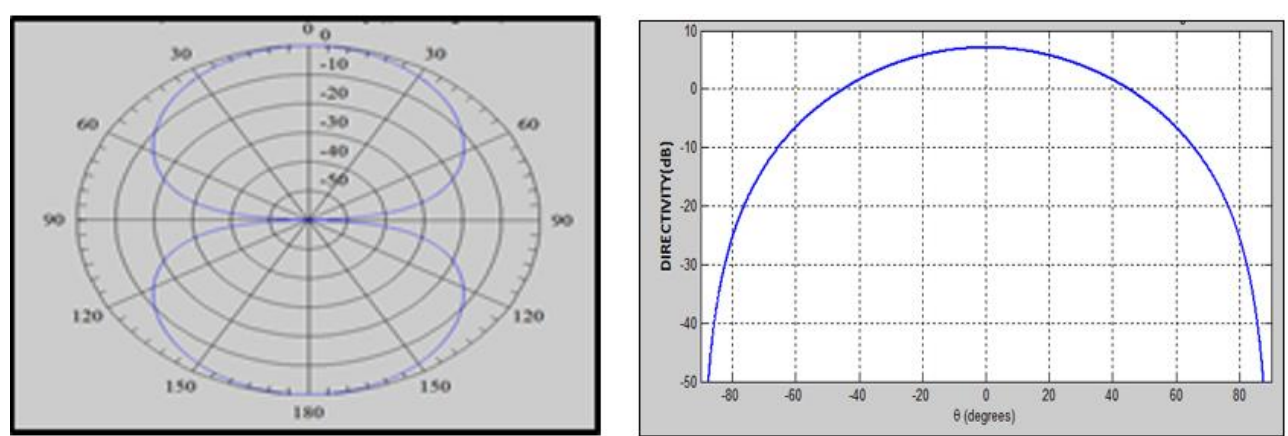

(a)
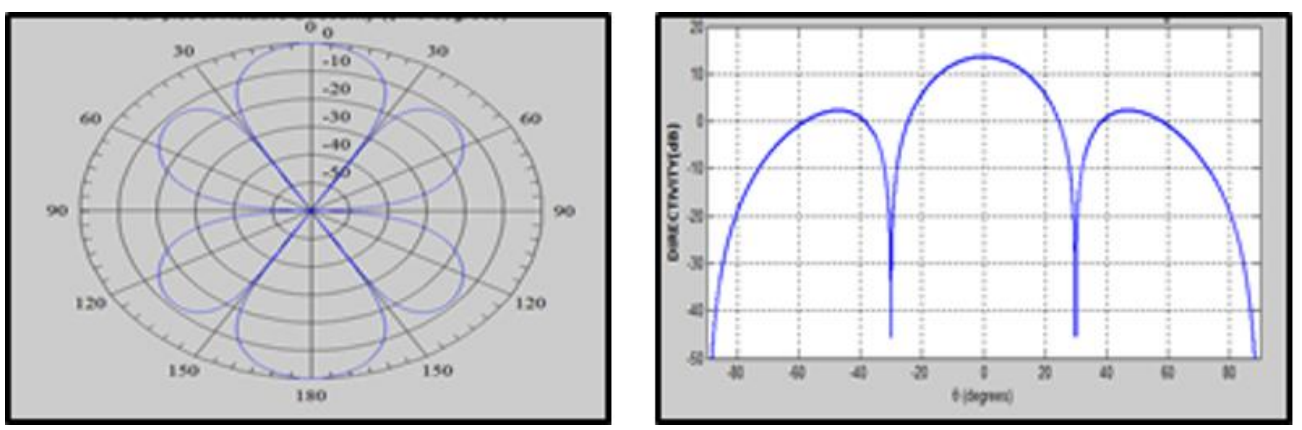

(b) 

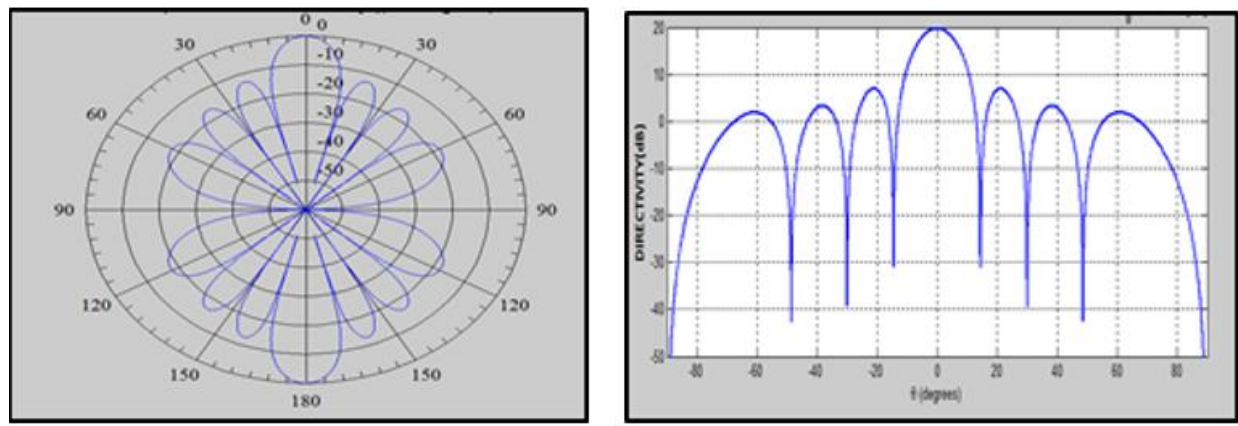

(c)
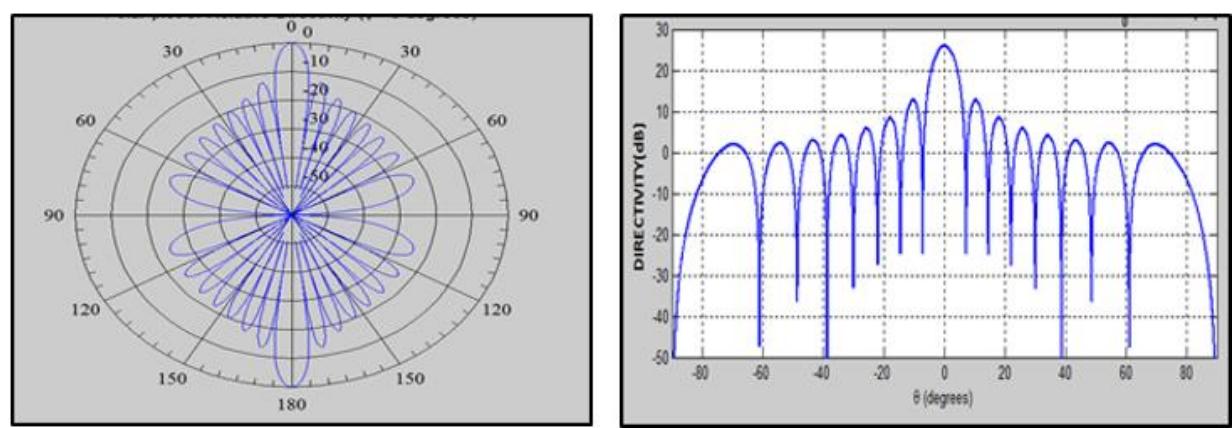

(d)

Figure 3. 2D, polar and linear plots of an antenna pattern with NXN planar arrays of isotropic radiators with a spacing of $\mathrm{dx}=\mathrm{dy}=\lambda / 2$, and equal amplitude and phase excitations

a) $2 \times 2$ b) $4 \times 4$ c) $8 \times 8$ d) $16 \times 16$

Figure 3 shows that starting from a unit array of $2 \times 2$, the pattern becomes more directive. The first unit array of 2x2 doesn't show any side lobe but constructing $4 \times 4$ rectangular array, two side lobes suddenly appeared. Similarly, as we increase the modular array i.e. $2 \times 2,4 \times 4,8 \times 8,16 \times 16$, the number of sidelobes follow a specific pattern as $0,2,6$, and 14 respectively. Therefore, the plotted array factor patterns clearly shows that the number of sidelobe for any $\mathrm{NxN}(2,4,8,16 \ldots)$ rectangular array can be easily predicted from the smallest $2 \times 2$ planar array. The combined for all planar array is shown in Figure 4.

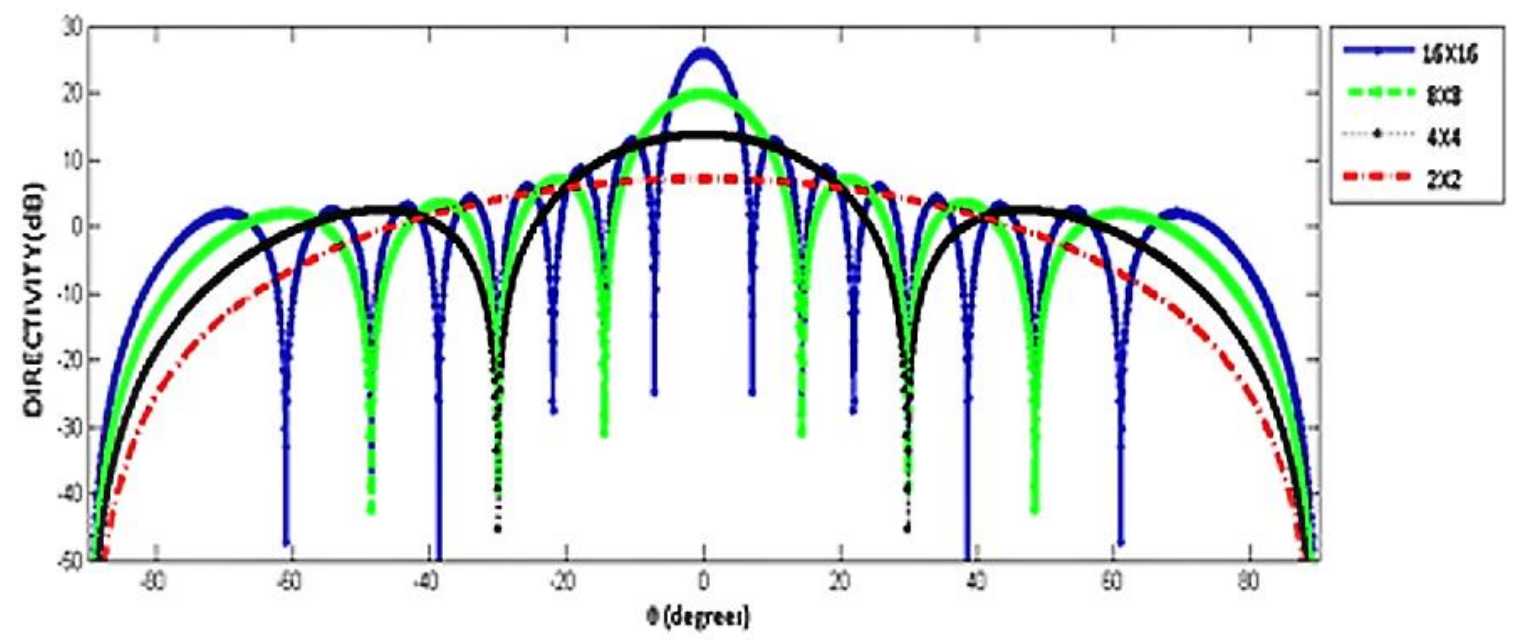

Figure 4. Combined array factor plots for $2 \times 2,4 \times 4,8 \times 8$, and $16 \times 16$ planar arrays 


\section{MODULARITY ANALYSIS OF SUB-ARRAYED PLANAR ARRAYS}

In this section, it is explained the results of research and at the same time is given the comprehensive discussion. Results can be presented in figures, graphs, tables and others that make the reader understand easily $[3,6]$. The discussion can be made in several sub-chapters.

\subsection{Matlab analysis}

Based on the array factor characteristics presented in Figure 3 and using (1-7), the directivity, gain, $3 \mathrm{~dB}$ half-power beams widths, no of sidelobes and sidelobe levels are calculated to analyse the modularity of sub-array. The behaviour of modular planar arrays are closely observed to derive the impact of placing planar antenna arrays in modular form i.e. $2 \times 2,4 \times 4,8 \times 8$ etc. The directivity, $3 \mathrm{~dB}$ half-power beam widths, number of sidelobes appearing and as well as the sidelobe level variations are presented in Table 1 and Figures 5 and 6.

Table 1. Comparison of directivity, gain, hpbw, number of sideslobes and sidelobe level variations

\begin{tabular}{cccccc}
\hline No Array elements & Directivity $(\mathrm{dB})$ & Gain $(\mathrm{dB})$ & 3dB HPBW (degrees) & No. of side lobes & Side lobe level $(\mathrm{dB})$ \\
\hline $2 \times 2$ & 7.06 & 6.64 & 60.33 & 0 & \\
$4 \times 4$ & 13.48 & 12.67 & 26.47 & 2 & -11.3 \\
$8 \times 8$ & 19.71 & 18.53 & 12.87 & 6 & -12.8 \\
$16 \times 16$ & 25.88 & 24.33 & 6.39 & 14 & -13.15 \\
$32 \times 32$ & 32.11 & 30.18 & 3.19 & 30 & -13.24 \\
$64 \times 64$ & 38.84 & 6.51 & 1.60 & 62 & -13.26 \\
\hline
\end{tabular}

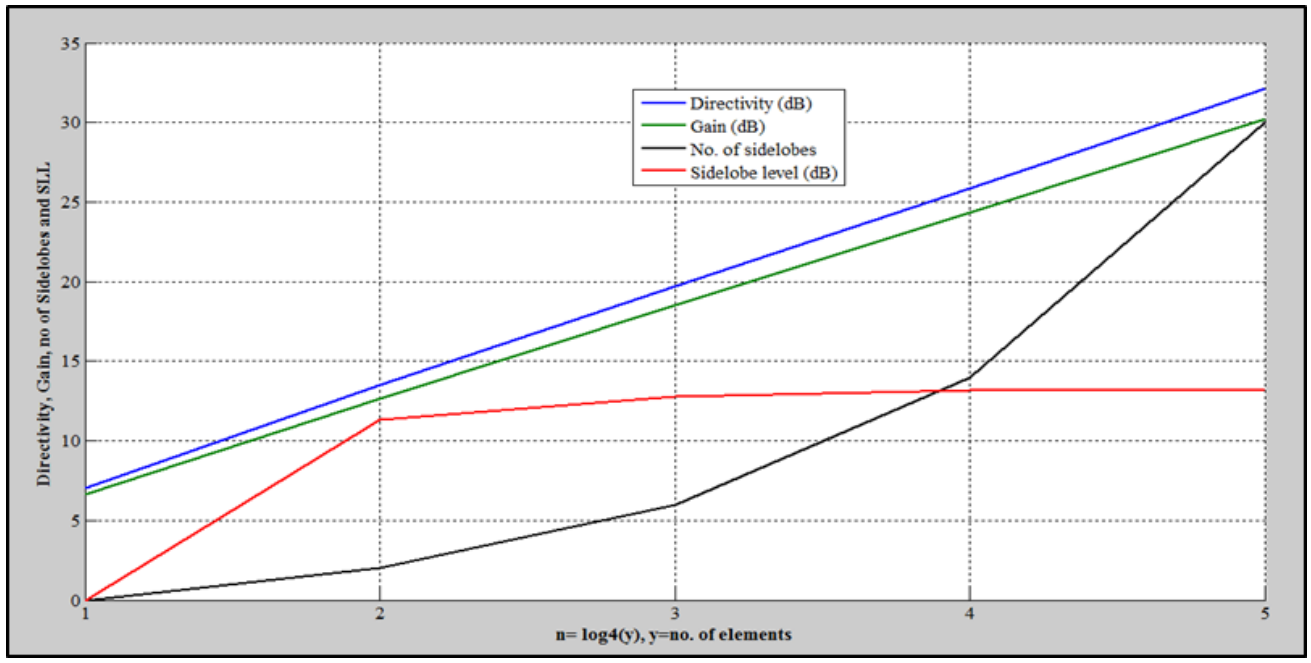

Figure 5. Variation of directivity, gain, no. of side lobes, and side lobe levels Vs no. of elements

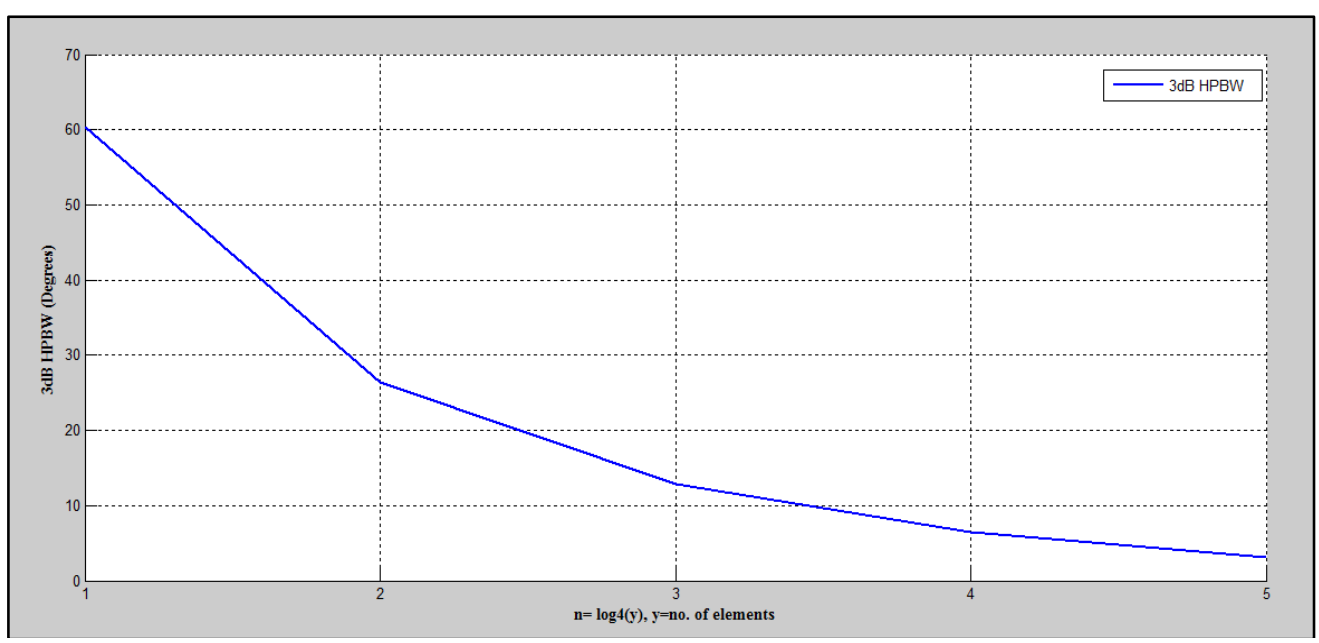

Figure 6. 3dB half-power beam width Vs no. of elements 
Referring to Table 1, planar antenna arrays has shown similar repeating radiation characteristics when they are placed in a modular form. It can be observed some similarities in their directivities, half-power beam widths and sidelobes. To start with directivity, the directivity increases by $6 \mathrm{~dB}$ every time when the array size is increased by a factor of 4 such as $2 \times 2,4 \times 4,8 \times 8,16 \times 16$ and $32 \times 32$. For example, $2 \times 2$ planar array has a directivity of $7.06 \mathrm{~dB}$ while it has increased to $13.48 \mathrm{~dB}$ in the case of $4 \times 4$ array and so on. Similarly, the gain of the array antennas follows the directivity accordingly when an antenna with efficiency of 0.94 was used. Thus, it can be concluded that there is $6 \mathrm{~dB}$ directivity and gain increase when the array enlarges along the given modular form.

Moreover, the half power beam-width is exponentially decreasing with the increase of array size. The decrease in half-power beam width is indirectly revealing the increase of that of directivity as the array becomes larger. The $3 \mathrm{~dB}$ HPBW variation shows a halving trend where it drops half every time when array size is increased modularly. For instance, $3 \mathrm{~dB}$ HPBW's for $2 \times 2,4 \times 4,8 \times 8$ planar arrays is $60.33,26.47$, and 12.87 degrees respectively.

From Figure 5, it is clearly seen that the directivity and gain are linearly increasing with the increase of number of elements in the array. Similarly, sidelobe level variations also increase linearly in the beginning before it becomes constant. But the number of sidelobes and $3 \mathrm{~dB}$ half power beam widths follow an exponential form where the directivity and HPBW are inversely related as illustrated in Figure 6.

\subsection{CST analysis}

Previously, Matlab software was used to analyse the radiation characteristics of a rectangular isotropic planar arrays to observe the effect of developing large arrays by repeating a small rectangular subarray many times placed in a modular form i.e. $2 \times 2,4 \times 4,8 \times 8,16 \times 16$ etc. It was found that those isotropic planar arrays has shown similar repeating radiation characteristics in their gain, directivities, half-power beam widths and sidelobes. Moreover, a mathematic model was derived to predict the radiation performance of large-scale arrays from the small sub-array.

Therefore, in order to validate those findings, CST software package is used to simulate planar of patch antennas for validation. In this part, the practical antenna effects such as mutual coupling between elements will be considered. Therefore, the results from the Matlab and CST will be compared in order to validate the derived model to predict the radiation performance of large arrays such as array factors, directivities, gains, half power beam-widths, sidelobe ratios and so on.

A single rectangular array was constructed and showed in Figure 7. The antenna is designed using Rogers RT5880 (lossy) dielectric substrate having with a dielectric constant is 2.2 and a thickness of $1 \mathrm{~mm}$. The feed line is a $50 \Omega$ microstrip line attached to the patch. The substrate is fully grounded. The operating frequency is set to $6 \mathrm{GHz}$. The behaviour of planar arrays are observed in $2 \times 2,4 \times 4$ and $8 \times 8$ on some specific array characteristics such as the directivity, gain, $3 \mathrm{~dB}$ half-power beam widths, number of sidelobes appearing and as well as the sidelobe level variations and Figure 8 represents the structure of planar arrays. The results are compared in tabulated form as shown in Table 2. hus, this analysis can be utilized to predict the radiation characteristics of large-scale arrays.

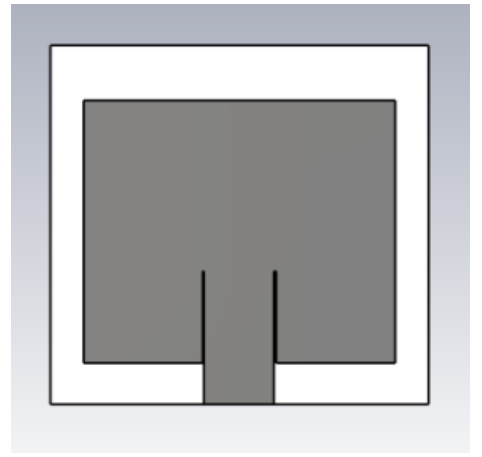

(a)

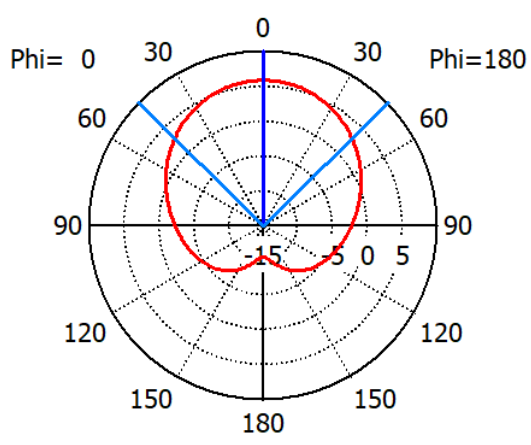

(b)

Figure 7. Single planar array results: (a) Structure, (b) Pattern 


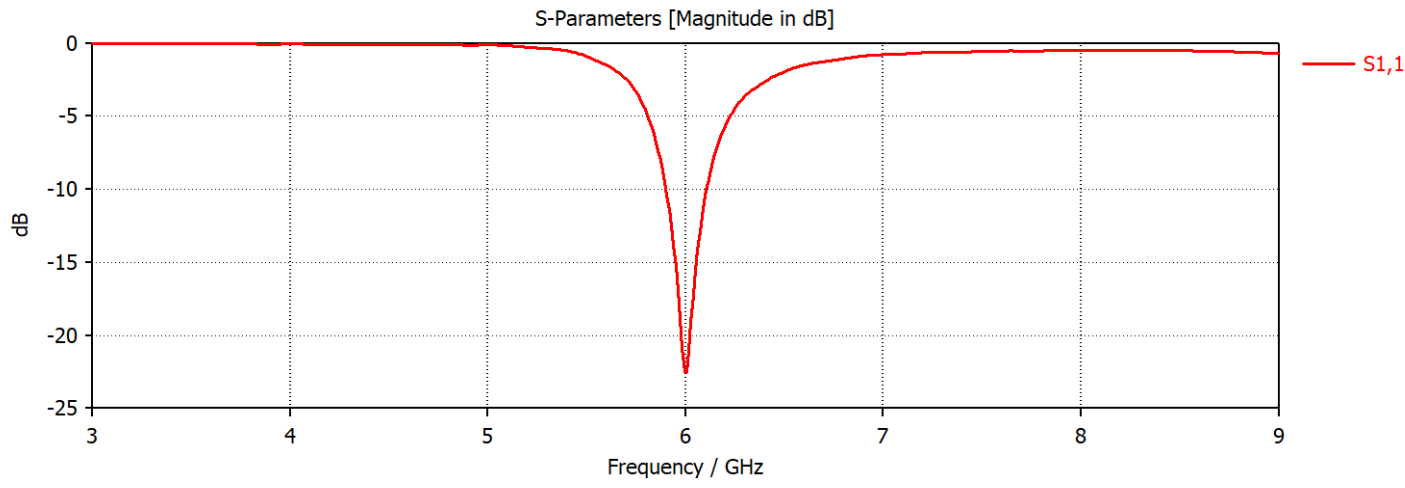

(c)

Figure 7. Single planar array results: (c) Sparameter

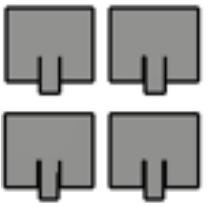

(a)

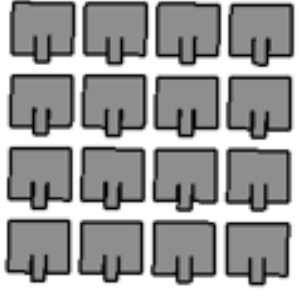

(b)

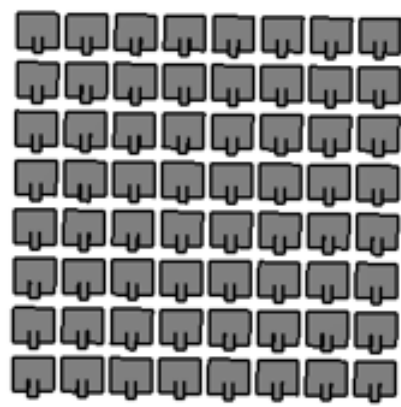

(c)

Figure 8. Structure of planar arrays, (a) $2 \times 2$, (b) $4 \times 4$, (c) $8 \times 8$

Referring to Table 2, after comparing theoritical calculation results and simulation results it can be found some connections between them. In terms of directivity the simulated results are minimum $4 \mathrm{~dB}$ higher than the calculated results. Moreover in the simulation results for $2 \times 2,4 \times 4$ and $8 \times 8$ almost $6 \mathrm{~dB}$ increase of directivity can be observed. In terms of gain the simulated results are also higher than the calculated results. For $3 \mathrm{~dB}$ HPBW the degrees drop in calculated results are much drastric than the simulated results. However, number of sidelobes are same in calculated resuls and simulated results. Table 3 illustrate 2D radiation pattern of theoretical calculation and simulation results. The similatiries between theoretical and simulation are clearly visible as the main lobes of $2 \times 2,4 \times 4$ and $8 \times 8$ are more likely similar and shoed the similar number of sidelobes.

Table 2. Comparison of directivity, realized gain, $3 \mathrm{~dB}$ angular width, number of sideslobes and Sidelobe Level Variations (CST)

\begin{tabular}{|c|c|c|c|c|c|c|c|c|c|c|}
\hline \multirow[b]{2}{*}{$\begin{array}{c}\text { Array } \\
\text { element } \\
\text { s }\end{array}$} & \multicolumn{2}{|c|}{ Directivity (dB) } & \multicolumn{2}{|c|}{ Realized Gain (dB) } & \multicolumn{2}{|c|}{ HPBW (degrees) } & \multicolumn{2}{|c|}{ No. of sidelobes } & \multicolumn{2}{|c|}{ sidelobe level $(\mathrm{dB})$} \\
\hline & $\begin{array}{c}\text { Calculate } \\
\text { d } \\
\text { (Matlab) }\end{array}$ & $\begin{array}{c}\text { Simulate } \\
\mathrm{d} \\
(\mathrm{CST})\end{array}$ & $\begin{array}{c}\text { Calculate } \\
\mathrm{d} \\
\text { (Matlab) }\end{array}$ & $\begin{array}{c}\text { Simulate } \\
\mathrm{d} \\
(\mathrm{CST})\end{array}$ & $\begin{array}{c}\text { Calculate } \\
\text { d } \\
\text { (Matlab) }\end{array}$ & $\begin{array}{c}\text { Simulate } \\
\mathrm{d} \\
(\mathrm{CST})\end{array}$ & $\begin{array}{c}\text { Calculate } \\
\text { d } \\
\text { (Matlab) }\end{array}$ & $\begin{array}{c}\text { Simulate } \\
\mathrm{d} \\
(\mathrm{CST})\end{array}$ & $\begin{array}{c}\text { Calculate } \\
\text { d } \\
(\text { Matlab) }\end{array}$ & $\begin{array}{c}\text { Simulate } \\
\mathrm{d} \\
(\mathrm{CST})\end{array}$ \\
\hline $2 \times 2$ & 7.06 & 12.6 & 6.64 & 8.94 & 60.33 & 29.8 & 0 & 0 & & -13.7 \\
\hline $4 \times 4$ & 13.48 & 17.7 & 12.67 & 13.7 & 26.47 & 23.4 & 2 & 2 & -11.3 & -14.6 \\
\hline $8 \times 8$ & 19.71 & 23.1 & 18.53 & 19 & 12.87 & 13.6 & 6 & 6 & -12.8 & -13.2 \\
\hline
\end{tabular}


Table 3. Comparison in term of 2D radiation pattern between theoritical calculation and simulation

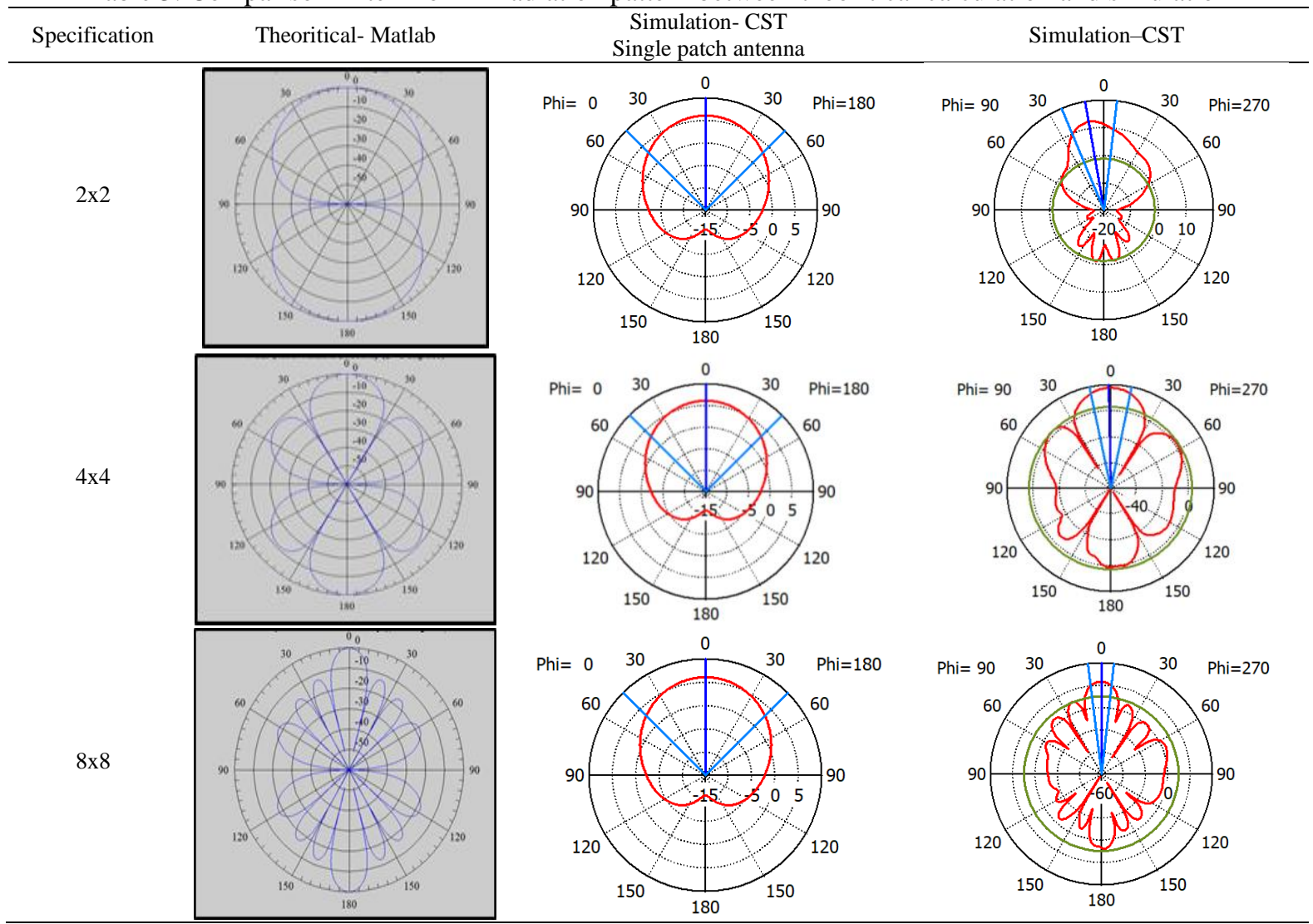

\section{CONCLUSION}

Large-scale antenna arrays have been concentrated to assess the possibility of a basic and productive method of utilizing sub-arrays for the improvement of expansive uniform arrays. Large arrays can be fashioned if small sub-arrays are repeated throughout the remaining large array. The antenna sub-array is examined based on the pattern, the directivity and the gain, 3dB half-power beam width, number of sidelobes and also the side-lobe level. There is $6 \mathrm{~dB}$ increase in gain and directivity when the array is enlarged along with the given modular form. The modularity is maintained in the CST simulations of the sub-arrays and validate the matlab analysis. By increasing the size of the array the half power beam-width decreases exponentially. The variations caused by the level of the side-lobes also escalates linearly at first before becoming constant. But the quantity of side-lobes and $3 \mathrm{~dB}$ half power beam widths follow an exponential method. So it can be concluded that, this analysis can be used to calculate the radiation characteristics of large-scale arrays by forming a small sub-array of $2 \times 2$ components as a perspective.

\section{ACKNOWLEDGEMENTS}

This work is partially funded by International Islamic University Malaysia (IIUM) Publication RIGS grant no. P-RIGS19-003-0003

\section{REFERENCES}

[1] AHM Zahirul Alam, Islam Md. Rafiqul, Khaizuran Abdullah, Eid Osman, Noor Hidayah M Adnan, Naimul Mukit , Rauful Nibir (2018), "Scalability Analysis for Designing Large-Scale Antenna Array Using Sub-Array", 7th International Conference on Computer and Communication Engineering (ICCCE), pp. 29 - 33. 2018.

[2] Noor Hidayah Muhamad Adnan, Islam Md. Rafiqul and AHM Zahirul Alam,"Massive MIMO for Fifth Generation (5G): Opportunities and Challenges", Proceedings of 6th International Conference on Computer and Communication Engineering, Kuala Lumpur, pp. 47-52, July 25-27, 2016,.

[3] N. H. M. Adnan, I. M. Rafiqul and A. H. M. Z. Alam, "Effects of inter element spacing on large antenna array characteristics," 2017 IEEE 4th International Conference on Smart Instrumentation, Measurement and Application (ICSIMA), Putrajaya, 2017, pp. 1-5. 
[4] W. L. Stutzman and G. A. Thiele, Antenna Theory and Design. New York: John Wiley \& Sons, 2012.

[5] E. Yaacoub, M. Al Husseini, A. Chehab, A. El Hajj, K. Y. Kabalan, "Hybrid Linear and Circular Antenna Arrays", Iranian Journal of Electrical and Computer Engineering, vol. 6, no. 1, pp. 48-54, WinterSpring 2015.

[6] A. J. Roscoe and R. A. Perrott, "Large finite array analysis using infinite array data," in IEEE Transactions on Antennas and Propagation, vol. 42, no. 7, pp. 983-992, July 1994.

[7] Balanis A. Constantine, Antenna Theory-Analysis and Design, 4th Edition, John Wiley \& Sons Inc, 2016.

[8] Ahmed A. Kishk," Prediction of Large Array Chracteristics from Small Array Parameters," 2nd European Conference on Antennas and Propagation UK, 11-16 Nov. 2012.

[9] W. Hong, K. Baek, Y. Lee, Y. Kim and S. Ko, "Study and prototyping of practically large-scale mmWave antenna systems for 5G cellular devices," in IEEE Communications Magazine, vol. 52, no. 9, pp. 63-69, September 2014.

[10] E. G. Larsson, O. Edfors, F. Tufvesson and T. L. Marzetta, "Massive MIMO for next generation wireless systems," in IEEE Communications Magazine, vol. 52, no. 2, pp. 186-195, February 2014.

[11] Mohammed Zaid, Md Rafiqul Islam, Mohamed H Habaebi, AHM Zahirul Alam, Khaizuran Abdullah, (2017). "Optimum concentric circular array antenna with high gain and side lobe reduction at $5.8 \mathrm{GHz}$ ", Proceedings of 6th International Conference on Mechatronics (ICOM2017), Kuala Lumpur, Malaysia, August 8-9, 2017.

[12] Noor Hidayah Muhamad Adnan, Islam Md. Rafiqul, AHM Zahirul Alam, "Effects of Inter-Element Spacing and Number of Elements on Planar Array Antenna Characteristics". Indonesian Journal of Electrical Engineering and Computer Science (IJEECS), April 2018, Vol. 10 No. 1, pp230-240.

[13] A. S. Osman, M. R. Islam and M. H. Habaebi, "Modeling of Multiband/Wideband Stack Series Array Antenna Configuration for 5G Application," 2016 International Conference on Computer and Communication Engineering (ICCCE), Kuala Lumpur, 2016, pp. 349-354.

[14] I. M. Rafiqul, R. Nibir, N. Mukit, S. O. Abdinasir, M. H. Habaebi and S. Yasmin, "Dual Band Antenna Design Using Stacked Series Array For Ka-Band Application," 2018 7th International Conference on Computer and Communication Engineering (ICCCE), Kuala Lumpur, 2018, pp. 114-118.

[15] S. B. Patil, R. D. Kanphade and V. V. Ratnaparkhi, "Design and performance analysis of inset feed microstrip square patch antenna for $2.4 \mathrm{GHz}$ wireless applications," 2015 2nd International Conference on Electronics and Communication Systems (ICECS), Coimbatore, 2015, pp. 1194-1200. 